\title{
ANÁLISE DE IMPACTOS AMBIENTAIS ATRAVÉS DE GEOTECNOLOGIAS EM ÁREA ÚMIDA DO MUNICÍPIO DE BONITO - MS, BRASIL
}

Helen Rezende de Figueiredo ${ }^{1 *}$, Darlene Gris ${ }^{1,2}$, Fabricio Bau Dalmas ${ }^{3}$, Luciano Furtado Loubet ${ }^{4}$, Antonio Conceição Paranhos Filho ${ }^{5}$

${ }^{1}$ Programa de Pós-Graduação em Tecnologias Ambientais, Laboratório de Geoprocessamento para Aplicações Ambientais, Faculdade de Engenharias, Arquitetura e Urbanismo e Geografia, Universidade Federal de Mato Grosso do Sul, Campo Grande, MS,

Brasil. helenrezende.bio@gmail.com

${ }^{2}$ Grupo de Pesquisas em Ecologia Florestal, Instituto de Desenvolvimento Sustentável Mamirauá, Tefé, AM, Brasil;

${ }^{3}$ Universidade UNG. Programa de Mestrado em Análise Geoambiental. Praça Tereza Cristina, n²39, Guarulhos - SP, Brasil;

${ }^{4}$ Ministério Público do Estado de Mato Grosso do Sul, Promotor de Justiça de Campo Grande - MS, Brasil;

5Laboratório de Geoprocessamento para Aplicações Ambientais, Faculdade de Engenharias, Arquitetura e Urbanismo e Geografia, Universidade Federal de Mato Grosso do Sul, Campo Grande, MS, Brasil.

Recebido em: 15/02/2020 - Aprovado em: 15/03/2020 - Publicado em: 30/03/2020 DOI: 10.18677/EnciBio 2020A2

As áreas úmidas são ambientes com grande biodiversidade e ecossistemas importantes para a manutenção de serviços básicos ao ambiente, como na filtragem da água que realiza recarga hídrica, funcionando como os rins dos ecossistemas aquáticos. As zonas úmidas são ambientes frágeis que estão passando por alterações ambientais que reduzem suas áreas. As geotecnologias vêm auxiliando no monitoramento dessas áreas. O objetivo desse estudo foi a identificação e análise de impactos ambientais em áreas úmidas através do sensoriamento remoto, utilizando o realce de imagens e análise multitemporal. Foi realizada a análise multitemporal através de imagens de satélites de 1984 a 2018 e empregado o realce de composição de bandas falsa-cor RGB para a fotointerpretração das cenas. A extração de bacia hidrográfica foi realizada com imagens SRTM gerada pelo GRASS. Através da extração de bacias foi verificado que as áreas úmidas pertencem a nascestes de dois grandes rios da região. Houve alterações ambientais principalmente a redução das áreas úmidas e expansão das atividades antrópicas de agricultura.

PALAVRAS-CHAVE: análise multitemporal, alterações antrópicas, sensoriamento remoto.

\section{ANALYSIS OF ENVIRONMENTAL IMPACTS IN A WETLAND USING GEOTECHNOLOGIES}

\section{ABSTRACT}

Wetlands are environments with great biodiversity and important ecosystems for the maintenance of basic services to the environment, as in the filtration of water that performs water recharge, functioning as the kidneys of aquatic ecosystems. Wetlands are fragile environments that are undergoing environmental changes that 
reduce their areas. Geotechnologies have been helping to monitor these areas. The objective of this study was the identification and analysis of environmental impacts in humid areas through remote sensing, using image enhancement and multitemporal analysis. Multitemporal analysis was carried out using satellite images from 1984 to 2018 and the use of RGB false-color band enhancement was used for the photointerpretation of the scenes. The hydrographic basin extraction was performed with SRTM images generated by GRASS. Through the extraction of basins, it was verified that the wetlands belong to the headwaters of two large rivers in the region. There have been environmental changes, mainly the reduction of wetlands and expansion of anthropic agricultural activities.

KEYWORDS: multitemporal analysis, anthropic changes, remote sensing.

\section{INTRODUÇÃO}

As áreas úmidas são ecossistemas muito importantes devido as suas funções fundamentais para a manutenção da vida. Contribuindo, principalmente, na recarga hídrica de aquíferos e lençol freático durante o ano, retenção de sedimento, filtragem da água, conservação do solo, regulagem do microclima, manutenção da biodiversidade entre outros (JUNK et al., 2014; CUNHA et al., 2015). Além disso, apresentam uma grandeza de biodiversidade intrínseca de cada região onde estão inseridas, exaltando ainda mais a importância dessas áreas (JUNK et al., 2006; JUNK et al., 2014).

Esses importantes ecossistemas são altamente vulneráveis a interferência humana (HU et al., 2017; MOLLES; SHER, 2019), e as principais perdas e degradações em áreas úmidas são frequentemente causadas pela atividade antrópica, tais como a transformação destas áreas úmidas em áreas agrícolas ou pastagens, de maneira que as consequências destas transformações podem desencadear contaminação de corpos de água por insumos agrícolas; erosão do solo; compactação do solo pelo pisoteio de animais de criação; retificação e canalização de rios; e falta de planejamento nas construções de rodovias e barragens (CUNHA et al., 2015). A degradação, mesmo que de um pequeno habitat, contribui para a diminuição do número de espécies (VIEIRA et al., 2018), pois esses organismos que são limitados a áreas pequenas possuem restrição de habitats e sua função ecológica, os tornando muito vulneráveis a extinção (MOLLES; SHER, 2019). Acrescentando assim, mais fatores para a necessidade da preservação desses locais.

Porém, a dificuldade na delimitação de áreas úmidas é recorrente, em muitos casos essas áreas são praticamente inacessíveis, o que reforça a necessidade do auxílio das geotecnologias para tornar mais viável a identificação dessas zonas. Entre essas o sensoriamento remoto é uma ferramenta eficaz e eficiente para detectar áreas de corpos hídricos e áreas úmidas em uma grande área (SILVA et al., 2008; GUO et al., 2017).

Existem várias técnicas de sensoriamento remoto, entre estas o realce de imagens multiespectrais, que analisa a refletância das bandas (LYON, 2001; WU, 2017). O sensor multiespectral captura as ondas refletidas ou emitidas do espectro eletromagnético do visível e do comprimento de onda mais longo, os infravermelhos próximo, médio e longo. O comportamento do espectro infravermelho próximo é majoritariamente absorvido pela água e corpos hídricos. Com a presença de clorofila os corpos hídricos absorvem em menor proporção o infravermelho, contudo as ondas mais curtas do azul ao violeta e o verde são absorvidos mais fortemente (PURKIS; ROELFSEMA, 2015; AMANI et al., 2018). 
Melo et al., (2017) realizaram um estudo de fotointerpretação de imagens de satélite através da composição de bandas falsa-cor RGB, identificando e quantificando cinco classes temáticas (corpos hídricos, Floresta Arbustiva, Herbácea/pastagem, Área Urbana e Cultura). Em outro estudo utilizando técnicas de cálculo entre as bandas do vermelho e infravermelho próximo para realizar o índice de vegetação por diferença normalizada (NDVI) e também utilizando a classificação automática não supervisionada, observou-se a perda de vegetação do tipo arbóreaarbustiva, em 2003 havia 59\% da vegetação em todo o Pantanal e em 2010 esse valor caiu para $42 \%$ dessa vegetação (PARANHOS FILHO et al., 2014). Assim, o objetivo desse trabalho foi a identificação e análise de impactos ambientais em áreas úmidas através do sensoriamento remoto, utilizando o realce de imagens e análise multitemporal.

\section{Área de estudo}

\section{MATERIAL E MÉTODOS}

Esse trabalho foi realizado no Município de Bonito, apresenta uma área de $4.934,414 \mathrm{~km}^{2}$ (IBGE, 2019), localizado no Estado de Mato Grosso do Sul. Este é um importante polo do ecoturismo no estado, conhecido internacionalmente, e referência em atividade de ecoturismo de baixo impacto ambiental.

A área do estudo está localizada a Noroeste do município, ao lado da Serra da Bodoquena que assinala a transição da Bacia do Paraná e Bacia do Paraguai (Figura 1), apresenta um clima tropical úmido. A Serra da Bodoquena é conhecida pelo terreno cárstico, formado por rochas carbonáticas (cálcio e dolomita) (DIAS, 2000; SALLUN FILHO; KARMANN, 2007). Os tipos de solos encontrados na área da pesquisa são Terra Roxa estruturada similar Distrófico Latossólica com textura argilosa e muito argilosa. No encrave com a Serra da Bodoquena apresenta solo tipo Rendzina textura argilosa com afloramento rochoso ondulado (BRASIL,1982).

$\mathrm{Na}$ área de estudo encontra-se vegetação do tipo Floresta Estacional Decidual Submontanhosa (BRASIL,1982); Cerrado onde se encontram culturas temporárias e a área úmida em questão. A área úmida apresentava áreas de ocorrência de lagoas e outras áreas com o solo encharcado, no seu entorno apresenta plantação de soja e solo arado.

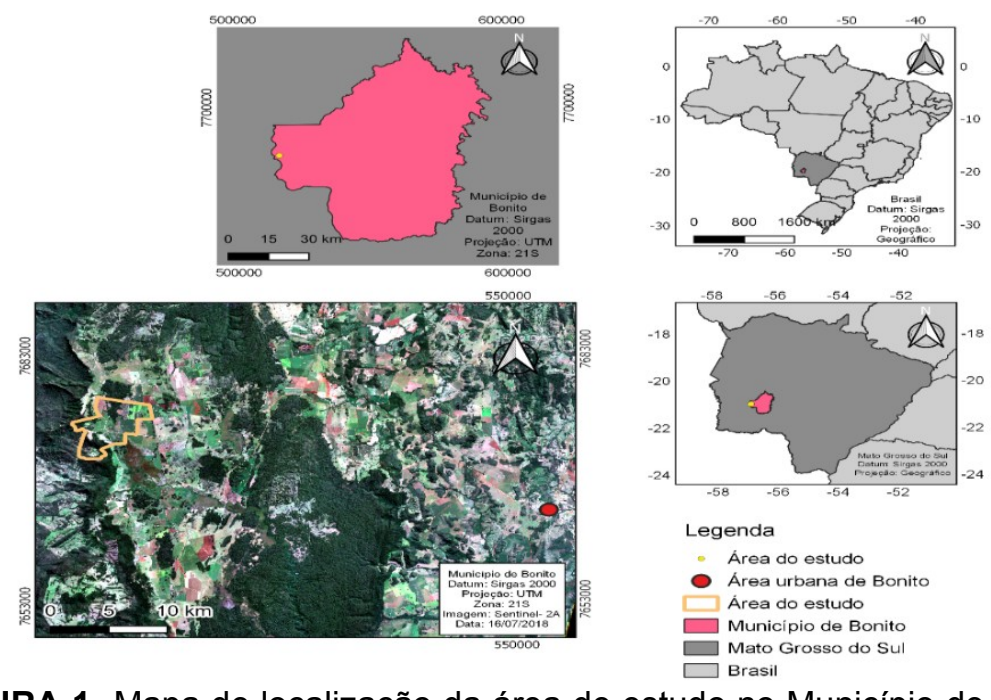

FIGURA 1- Mapa de localização da área de estudo no Município de Bonito.

Fonte: Elaborada pelos autores (2019). 
Aquisição das imagens de satélite

As imagens utilizadas no estudo foram adquiridas gratuitamente no site United States Geological Survey (USGS), missão EarthExplore (USGS, 2018a). Os satélites utilizados foram da missão Landsat, que apresentam imagens multiespectrais e resolução espacial de 30 metros, sensor Landsat 5 Thematic Mapper (TM) operando de 1984 a maio de 2012. Também foram utilizadas as imagens da missão Sentinel- 2A do Programa GMES (Global Monitoring for Environment and Security) sensor multi-spectral instrument (MSI) disponível desde junho de 2015, que apresentam maior resolução espacial de 10 e 20 metros, observa-se maior detalhamento das feições nas imagens. Todas as imagens foram obtidas sob demanda e apresentam correção atmosférica e calibração radiométrica, esses produtos são gerados no Earth Resources Observation and Science (EROS) e os dados de cálculo e algoritmo utilizados estão disponíveis no guia disponível pelo site USGS/Landsat (USGS, 2018b). Os critérios para seleção das imagens foram período de seca e com ausência de nuvens. No quadro 1, são mostradas as datas e os sensores utilizados.

QUADRO 1- Data de aquisição das imagens de satélites usadas no estudo.

\begin{tabular}{lll}
\hline Satélite/ Sensor & Órbita/Ponto & Data de passagem \\
\hline Landsat 5/ TM (USGS, 2018c) & $226 / 075$ & $16 / 07 / 1984$ \\
& & $07 / 08 / 1986$ \\
& & $13 / 08 / 2000$ \\
Sentinel- 2A/ MSI (USGS, 2018d) & T21KWS & $09 / 09 / 2016$ \\
\hline
\end{tabular}

Fonte: autores (2019).

Análise dos dados

Todos os processamentos de dados foram realizados no software QGIS, que é livre e com código aberto (QGIS DEVELOPMENT TEAM, 2018).

Foi realizado uma visita in loco em áreas com potencial a serem áreas úmidas. As inspeções em campo foram realizadas no dia 26 de abril de 2018.

\section{Delimitação de Bacia Hidrográfica}

Foi realizada a delimitação da bacia hidrográfica com o objetivo de identificar quais as bacias que pertenciam as áreas úmidas. Foi utilizada uma cena da imagem de radar da missão espacial Topográfica Radar Shuttle (SRTM), que consiste em imagem com dados de radar para coletar informações sobre a elevação terrestre. Foi adquirido também pelo site USSG/EarthExplore (USGS, 2018e). Foram delimitadas as microbacias e depois a drenagem dos rios através da ferramenta GRASS GIS (Geographic Resources Analysis Support System), presente no software QGIS. Segundo as informações do site (GRASS, 2019), a mesma utiliza o algoritmo $A^{\top}$ least-cost search descrita por Ehlschlaeger (1989).

Realce das imagens

Para a análise multitemporal por fotointerpretação, foi necessário realizar o processamento das bandas. As imagens adquiridas dos satélites estão separadas em diversas bandas, de acordo com o comprimento de onda de cada sensor. Para compor uma única cena multiespectral foi realizado o empilhamento destas bandas e feito de acordo com as características dos alvos estudados, sendo as áreas úmidas com lâmina de água, solo úmido com ou sem vegetação, lagoas e corpos hídricos. 
O realce tem o propósito de melhorar a qualidade visual da imagem, facilitando a análise do fotointérprete e enfatizar alguma característica de interesse para aplicação específica (MENESES; ALMEIDA, 2012). O realce por composição colorida no canal do RGB (Red, Green e Blue) é necessário para produzir a percepção visual, pois as imagens de satélite armazenam informações da refletância dos alvos na terra que é convertida em números, chamados Digital Numbers (DN), ou seja, valores digitais de brilho das bandas e registrada em diferentes níveis de cinza em cada pixel. Quanto maior a refletância do objeto, maior será o DN, que adquire uma tonalidade mais escura de cinza (IBGE, 2001; FLORENZANO, 2011; MENESES; ALMEIDA, 2012).

Através do contraste da reflectância espectral dos alvos a composição colorida realça cada alvo em diferentes cores e tonalidades. Assim é possível distinguir a água, pois esta absorve a maioria do comprimento de onda do visível e com maior intensidade o infravermelho próximo, obtendo tonalidades escuras. Já a vegetação reflete em maior intensidade os comprimentos de onda dos infravermelhos apresentando tonalidades mais claras e vivas (BARBOSA, 2019; NOVO et al., 2019).

A combinação das bandas nos canais RGB, são chamadas de falsa-cor colorida, neste trabalho optou-se por utilizar as bandas do infravermelho médio (SWIR1), infravermelho próximo (NIR) e vermelho (Red). Após a composição foi realizado o realce MinMax, que faz com que os valores dos pixels de cada banda sejam reescalados de acordo com os valores mínimos e máximos (IBGE, 2001). Segundo Meneses e Almeida (2012) esse procedimento mantem a base de dados normalizada à escala total do intervalo dinâmico de brilho.

A análise foi realizada através da fotointerpretação dos alvos de acordo com as características de cor, tonalidade, tamanho, sombreamento, textura, padrão, localização e forma (LILLESAND et al., 2004; FRITZ, 2008) referentes a cada alvo.

\section{RESULTADOS}

Através da delimitação de bacia por imagem de radar (SRTM) foi possível observar que o curso de água e as áreas úmidas são nascentes de duas microbacias: a sudeste a microbacia do Rio Perdido e a sudoeste a microbacia do Rio Aquidabã (Figura 2).

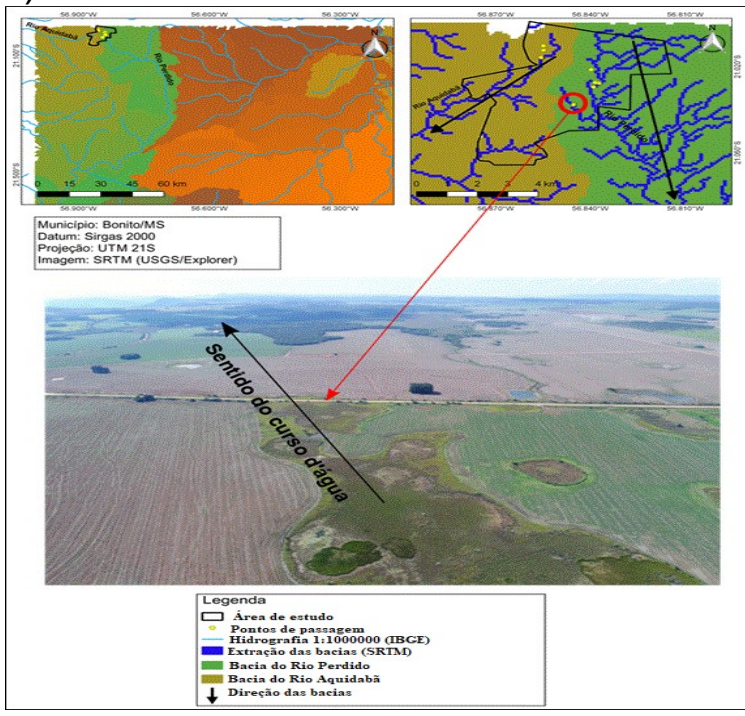

FIGURA 2- Imagem de SRTM (radar) utilizada para procedimento de delimitação de bacia mostrando que as áreas úmidas pertencem a duas microbacias: do Rio Aquidabã e do Rio Perdido. Fonte: Elaborada pelos autores (2018). 
O solo apresentava as seguintes características hidromórficas, gleissolo, preto acinzentado, que se encontrava bastante encharcado, inclusive com água corrente (Figuras 3), esses dados foram observados in loco. Apesar da vegetação estar descaracterizada, com plantas exóticas, ainda assim essas áreas apresentavam características (solo encharcado, espécies herbáceas características, entre outros) e local de ocorrência que indicam as mesmas serem áreas úmidas. Nas áreas úmidas foram encontrados drenos com profundidades variadas, além de áreas com solo escuro que apresentavam aspecto rachado, provavelmente em épocas de chuva esses locais ficam encharcados.

Em alguns locais, em meio a plantação, haviam cavidades com afloramentos rochosos na superfície constituindo um corpo hídrico (grotas) (Figura 3). Outro tipo de nascente foi constatada na área, formando lagos de coloração azul, estas nascentes foram observadas na visita a campo no dia 26 de abril de 2018.
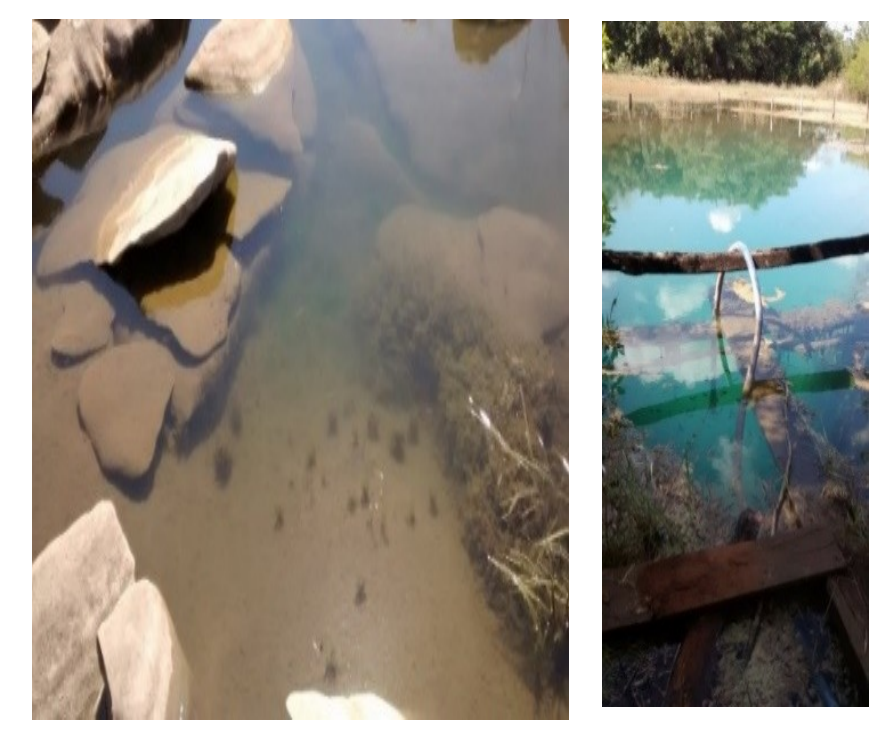

FIGURA 3- Nascentes: A- grota com afloramento rochoso, apresentando água turva; B- lago de coloração azul.

Fonte: Elaborada pelos autores (2018).

Através do realce falsa-cor pode-se constatar que no ano de 1984 ocorria uma extensa área úmida e a área de plantação se limitava a poucos hectares (cerca de 451,10 ha), também observou-se a presença de um canal no meio da lavoura (Figura 4). Este canal faz ligação com outra extensa área úmida que corre sentido sudeste e pertence a bacia hidrográfica do Rio Perdido. Na porção sudoeste, havia acúmulo de água, formando lagoas. 


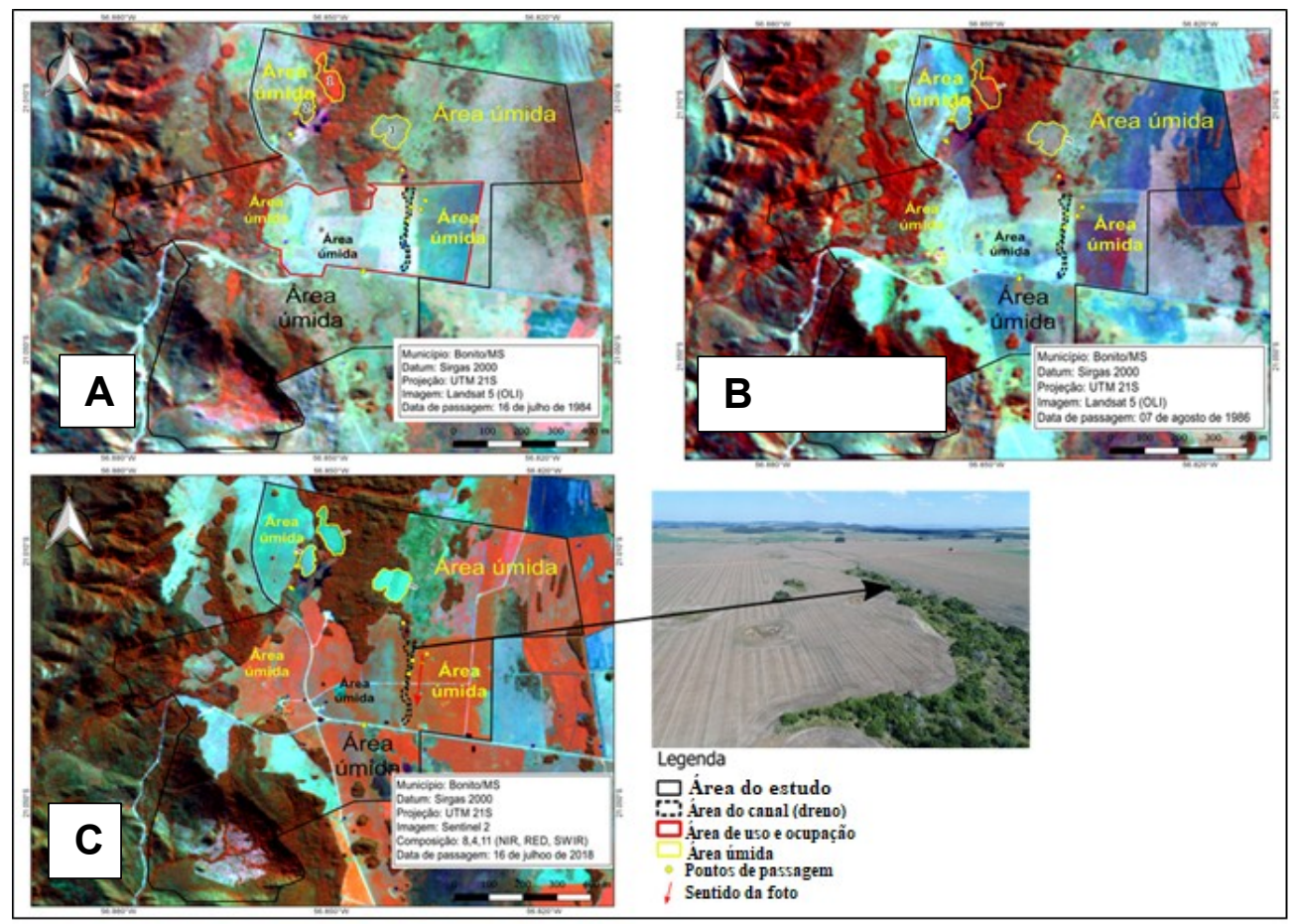

FIGURA 4- Imagens de satélites dos meses considerados secos mostrando a área em vários anos. A e B: imagens Landsat 5 de 1984 e 1986 (respectivamente) mostrando a extensão das áreas úmidas, lavoura e existência de um canal. C: Imagens Sentinel- 2A mostrando que as áreas úmidas foram substituídas por lavoura.

Fonte: Elaborada pelos autores (2018).

Após dois anos, em 1986, houve uma expansão expressiva da área de cultivo e as áreas úmidas foram reduzidas aos pontos com solos encharcados, o que provavelmente dificulta a entrada de máquinas para o plantio. Entre os anos 2000 a 2018, observa-se algumas áreas perto da mata que foram drenadas para realizar cultivo, estendendo a área de cultivo a cerca de 1306,66 ha (Figura 5).

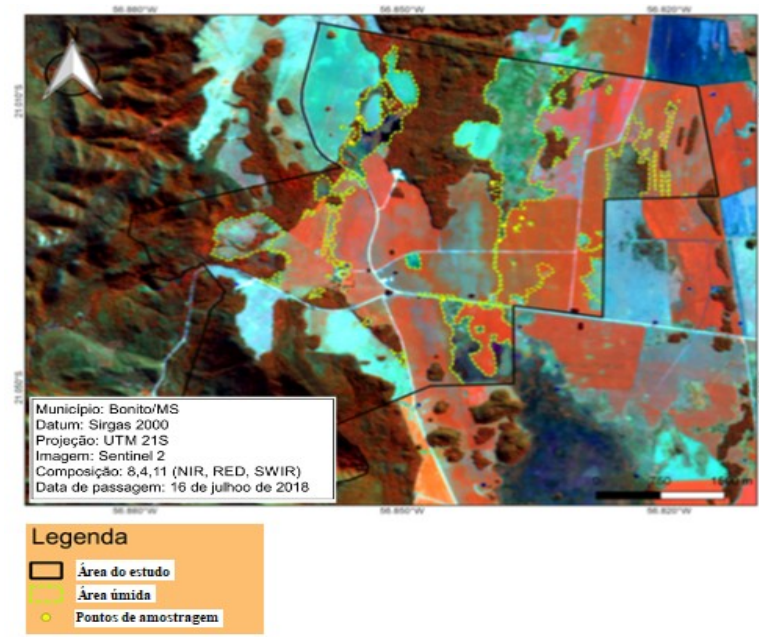

FIGURA 5- Imagens de satélites dos meses considerados secos mostrando a expansão da área para atividade de agricultura, no ano de 2018.

Fonte: Elaborada pelos autores (2018). 


\section{DISCUSSÃO}

Bonito é conhecido pela beleza cênica dos rios, um desses rios presentes no município é o Rio Perdido, que apresenta águas de cor azul-esverdeada e é importante na drenagem da Serra da Bodoquena. Segundo o Plano de Manejo da Serra da Bodoquena, essas áreas úmidas das nascentes do Rio Perdido são imprescindíveis para a conservação da carga hídrica da Serra da Bodoquena, essas áreas desempenham papel de filtragem, armazenar e abastecer os aquíferos (BRASIL, 2013).

As áreas úmidas analisadas, apresentam características hidrogeomorfológicas análogas às veredas, com solo preto acinzentado e vegetação típica de vereda, corroborando com os levantamentos realizados por outros estudos (BRASIL, 2013; SCREMIN-DIAS et al., 2018). As veredas são nascentes difusa, ou seja, a exfiltração não pode ser pontuada, não sendo possível afirmar com precisão o principal local de saída da água, formando terrenos de solo encharcado. O Código Florestal (Lei Federal n. 12.651/2012), no artigo $4^{\circ}, \mathrm{XI}$, determina como Área de Preservação Permanente (APP) na largura de 50 (cinquenta) metros a partir do espaço permanentemente brejoso e encharcado (BRASIL, 2012). O que não foi constatado em vários locais da área de estudo.

Essas áreas úmidas funcionam como rins que filtram material antes da água abastecer os corpos hídricos superficiais ou subterrâneos. As zonas úmidas são formações únicas que limpam sólidos e nutrientes da água no ambiente. Apresentam vegetações e animais adaptados a variação periódica do lençol freático. A redução das áreas úmidas tem inúmeras consequências ao longo dos anos, como a fragmentação e a ampliação da distância entre os corpos hídricos existentes, dificultando a capacidade de dispersão e de colonização de plantas e animais (JUNK et al., 2014; CUNHA et al., 2014; TINER, 2016).

$\mathrm{Na}$ área de estudo foi constatado outros tipos de corpos hídricos, as nascentes de fundo de vale que criam acúmulo inicial de água, comum quando há camada rochosa por baixo do lençol freático raso que acaba formando um lago (BARRETO et al., 2010). Já as grotas, que têm origens em rios subterrâneos, são nascentes pontuais, ou seja, são nascentes em que ocorre concentração da exfiltração em apenas um ponto, constituindo um fluxo único. As nascentes em rochas cársticas são originadas de dutos ou canais cársticos, resultado da dissolução (SÁNCHEZ; LOBO, 2016).

O solo encontrado na região apresenta textura argilosa e cor acinzentada. Segundo BRASIL (1982) neste local de estudo é encontrado o solo do tipo distrófico latossólico com textura muito argilosa. O solo tipo distrófico apresenta reserva de nutrientes restritiva, com isso a utilização agrícola com bons rendimentos ocorre nos primeiros anos e, posteriormente, apresenta um declínio rápido havendo necessidade de fertilizantes e corretivos no solo (CHAGAS et al., 2009). Foi verificado nas imagens multitemporais que essas áreas apresentam cultivo desde o ano 1984, o que justifica a coloração e a turbidez da grota encontrada em meio a plantação, sugerindo utilização de insumos agrícolas.

A redução da vegetação das áreas úmidas observada ao longo dos anos neste trabalho foi principalmente decorrente da drenagem para a atividade de agricultura, ocasionando a diminuição do volume de água e a fragmentação de habitats ao longo do rio. A perda de biodiversidade de plantas e animais desses ambientes pode acontecer com a redução das áreas úmidas, devido a especificidade de habitat desses organismos (BATZER; BOIX, 2016). Alterando também a forma, a sua área e processos ecológicos originais dos ecossistemas 
existentes (COLVIN et al., 2019). É uma constatação preocupante levando em consideração a diversidade de corpos hídricos e a reduzida extensão da área úmida que se mantem. Essa tendência de perda de vegetação nativa para atividades agrícolas pode ser observada no estudo de Silva e Pivello (2009) que apontaram o crescimento da agricultura de 9.651 ha no Município de Bonito entre os anos de 1986/1987 e 2004.

O expressivo avanço da agricultura pode ocasionar o aumento da vulnerabilidade do solo por processos erosivos, podendo resultar na intensificação de sedimentos carreados para rios, aumentando a turbidez e afetando a transparência das águas de Bonito, que é reconhecido internacionalmente (TERUYA JUNIOR et al., 2018). Segundo Chagas et al., (2009), áreas com presença de Gleissolo, típicos de áreas úmidas, devem ser preferencialmente destinadas para conservação devido à alta fragilidade ambiental pelas características dos solos, do relevo e da vegetação natural nessas terras. As áreas úmidas são ecossistemas sensíveis as ações antrópicas e necessitam de atenção no monitoramento de alterações ambientais para estratégias de controle e preservação desses remanescentes de áreas úmidas e nascentes na região de Bonito.

\section{CONCLUSÕES}

Foi observada a redução expressiva da área úmida para a atividade de cultura temporária, havendo descaracterização da vegetação natural e presença de inúmeros drenos. Demonstrando que essas áreas estão suscetíveis à perturbação humana, onde na verdade, deveria haver preservação, pela sua inquestionável função ecológica, cultural e social. Essas áreas úmidas são de alta fragilidade ambiental, principalmente por serem cabeceiras de importantes rios da região, o Rio Perdido e o Rio Aquidabã.

Através de técnicas consideravelmente simples de sensoriamento remoto como o realce de imagens nos canais RGB falsa-cor e análise multitemporal foi possível observar nitidamente a redução da área úmida e o aumento das ações antrópicas ao longo dos anos. Essas técnicas demonstram agilidade e fácil manuseio para a detecção de alterações ambientais em áreas de preservação, além de confirmar uma ferramenta para análise de extensas áreas com difícil acesso, como foi contatado no local.

\section{AGRADECIMENTOS}

O presente trabalho foi realizado com apoio da Fundação Universidade Federal de Mato Grosso do Sul - UFMS/MEC - Brasil, Programa de Pós-Graduação em Tecnologias Ambientais, Ministério Público do Estado de Mato Grosso do Sul e Promotoria de Justiça de Bonito. Agradecemos aos colegas do Laboratório de Geoprocessamento para Aplicações Ambientais pelo auxílio, a Coordenação de Aperfeiçoamento de Pessoal de Nível Superior (CAPES) pela disponibilização do Portal de Periódicos e ao Conselho Nacional de Desenvolvimento Científico e Tecnológico (CNPq) pela Bolsa de Produtividade em Pesquisa (Processo 305013/2018-1) de A.C. Paranhos Filho.

\section{REFERÊNCIAS}

AMANI, M.; SALEHI, B.; MAHDAVI, S.; GRANGER, J. Spectral analysis of wetlands using multi-source optical satellite imagery. ISPRS Journal of Photogrammetry and Remote Sensing, v.144, n.2018, p.119-136, 2018. Disponível em: https://www.researchgate.net/publication/326517742_Spectral_analysis_of_wetlands _using_multi-source_optical_satellite_imagery DOI: 10.1016/j.isprsjprs. $\mathbf{2 0 1 \overline { 8 }} .07 .005$. 
BARBOSA, C.C.F. Princípios físicos do sensoriamento remoto aquático. In: BARBOSA, C.C.F.; NOVO, E.M.L.M.; MARTINS, V.S. Introdução ao sensoriamento remoto de sistemas aquáticos: princípios e aplicações. INPE, São José dos Campos - SP, 2019. Disponivel em: http://www.dpi.inpe.br/labisa/livro/ Acessado em: 09/01/2020.

BARRETO, S. R.; RIBEIRO, S. A.; BORBA, M. P. Nascentes do Brasil: estratégias para a proteção de cabeceiras em bacias hidrográficas. WWF - BRASIL, São Paulo, $2010 . \quad$ Disponivel em: http://www.terrabrasilis.org.br/ecotecadigital/pdf/nascentes-do-brasil-estrategiaspara-a-protecao-de-cabeceiras-em-bacias-hidrograficas.pdf Acessado em: 16/06/2018.

BATZER, D.; BOIX, D. An introduction to freshwater wetlands and their invertebrates. In: Invertebrates in Freshwater Wetlands. Springer, Cham, p.1-23, 2016.

BRASIL. Instituto Chico Mendes de Conservação da Biodiversidade. Plano de manejo do parque serra da bodoquena. Brasília, 2013. Disponível em:http://www.icmbio.gov.br/portal/unidadesdeconservacao/biomasbrasileiros/ cerrado/unidades-de-conservacao-cerrado/2082-parna-da-serra-da-bodoquena Acessado em: 03/05/2018.

BRASIL. Ministério Das Minas E Energia Secretaria Geral. Folha SF. 21 Campo Grande: geologia, geomorfologia, pedologia, vegetação, uso potencial da terra/ Projeto RADAMBRASIL [v. 28]. Rio de Janeiro: O Projeto, p. 416, 1982. Disponível em: https://biblioteca.ibge.gov.br/index.php/biblioteca-catalogo? view=detalhes\&id=214553 Acessado em: 07/08/2017.

BRASIL. Lei Federal n. 12.651/2012, de 25 de maio de 2012. Presidência da República, Casa Civil: Subchefia para Assuntos Jurídicos, 2012. Disponível em: http://www.planalto.gov.br/ccivil_03/_ato2011-2014/2012/lei//12651.htm Acessado em: 20/01/2019.

CHAGAS, C. da S.; CARVALHO JUNIOR, W. de.; BHERING, S.B.; AMARAL, F.C.S. do; PEREIRA, N.R.; SILVA, E.F. da; et al.,; Zoneamento Agroecológico do município de Bonito/MS. Rio de janeiro: Embrapa Solos, p.66, 2009. Disponível em: https://www.infoteca.cnptia.embrapa.br/infoteca/handle/doc/696654 Acessado em: 07/08/2017.

COLVIN, S.A.R.; SULLIVAN, S.M.P.; SHIREY, P.D.; COLVIN, R.W.; WINEMILLER, K.O.; et al.; Headwater streams and wetlands are critical for sustaining fish, fisheries, and ecosystem services. Fisheries, v.44, n.2, p.73-91, 2019. Disponível em: https://afspubs.onlinelibrary.wiley.com/doi/abs/10.1002/fsh.10229 DOI: https://doi.org/10.1002/fsh.10229

DIAS, J. A região cárstica de Bonito, MS: uma proposta de zoneamento geoecológico a partir de unidades de paisagem. Ensaios e Ciência: Ciências Biológicas, Agrárias e da Saúde, v.4, n.1, p.9-43, 2000. Disponível em: https://www.redalyc.org/pdf/260/26040102.pdf Acessado em: 22/12/2019

EHLSCHLAEGER, C.R. Using the AT Search Algorithm to Develop Hydrologic Models from Digital Elevation Data. In Proceedings of the International Geographic Information System (IGIS), Baltimore, MD, p. 275-281, 1989. ENCICLOPÉDIA BIOSFERA, Centro Científico Conhecer - Jandaia-GO, v.17 n.31; p. 22 2020 
Disponível

em:

https://www.researchgate.net/publication/243781937_Using_the_AT_search_a gorithm_to_develop_hydrologic_models_from_digital_elevation_data Acesso em: $12 / 06 / 2 \overline{0} 18$

FLORENZANO T.G. Iniciação em sensoriamento remoto. Oficina de Textos, São Paulo - SP, 3 ed, 2011.

FRITZ, P. R. Geoprocessamento sem complicação. São Paulo: Oficina de textos, São Paulo - SP, 2008.

GRASS - Development Team (2017). Geographic Resources Analysis Support System livre e aberto. Calculates hydrological parameters and RUSLE factors. Disponível em: https://grass.osgeo.org/grass79/manuals/r.watershed.html Acesso em: $12 / 06 / 2018$

GUO, M.; L.I.J.; SHENG, C.; XU, J.; WU, L. A Review of Wetland Remote Sensing. Sensors (Basel). v.17, n.4, p.777, 2017. Disponível em: https://www.ncbi.nlm.nih.gov/pmc/articles/PMC5422050/ DOI:10.3390/s17040777

HU, S.; NIU, Z.; CHEN, Y.; LI, L.; ZHANG, H. Global wetlands: Potential distribution, wetland loss, and status. Science of the total environment, v.586, p.319-327, 2017. DOI: https://doi.org/10.1016/j.scitotenv.2017.02.001 Acessado em: 20/12/2019

IBGE. Instituto Brasileiro de Geografia e Estatística . Introdução ao processamento digital de imagens / IBGE, Primeira Divisão de Geociências do Nordeste (Manuais Técnicos em Geociências, n. 9). Rio de Janeiro: Fundação IBGE, n.9, p.94, 2001. Disponível em: https://biblioteca.ibge.gov.br/index.php/biblioteca-catalogo?view=detalhes\&id=2780 Acesso em: 22/08/2018

IBGE. Instituto Brasileiro de Geografia e Estatística O Cidades@. Disponível em: https://cidades.ibge.gov.br/brasil/ms/bonito/panorama Acessado em: 06/12/2019.

JUNK, W.J.; DA CUNHA, C.N.; WANTZEN, K.M.; PETERMANN, P.; STRÜSSMANN, C.; MARQUES, M.I.; AIS, J. Biodiversity and its conservation in the Pantanal of Mato Grosso, Brazil. Aquatic Sciences, v.68, p.278-309, 2006. DOI 10.1007/s00027-0060851-4 Acessado em: 12/12/2019

JUNK, W.J.; PIEDADE, M.T.F.; LOURIVAL, R.; WITTMANN, F.; KANDUS, P.; LACERDA, L.D.; et al.; Parte I: Definição e Classificação das Áreas Úmidas (AUs) brasileiras: Base Científica para uma Nova Política de Proteção e Manejo Sustentável. In Classificação e delineamento das áreas úmidas brasileiras e de seus macrohabitats. NUNES DA CUNHA, C.; PIEDADE, M. T. F.; JUNK, W. J. eds. Cuiabá: EdUFMT, 2014.

LILLESAND, T.M.; KIEFER, R.W.; CHIPMAN, J.W. Remote sensing and image interpretation. 5. ed. New York: J. Wiley, p. 763, 2004.

LYON, J.G. Wetland landscape characterization: techniques and applications for GIS, mapping, remote sensing, and image analysis. CPR Press; 1 edition, $p$. 160, 2001.

MELO, C.G.; LUZ, I.C.; OSCO, L.P.; RAMOS, A.P.M. Chave de interpretação para o mapeamento do uso e cobertura da terra da bacia hidrográfica do alto curso do rio 
santo Anastácio. Colloquium Exactarum, v.9, n. suplemento especial, p.226- 233, 2017. Disponível em: http://www.unoeste.br/site/enepe/2017/suplementos.aspx? area=Exactarum DOI: 10.5747/ce.2017.v9.nesp.000147

MENESES, P.R.; ALMEIDA, T. de (Org.). Introdução ao Processamento de imagens de sensoriamento remoto. Brasília: UNB/CNPq, 2012. 266 p. Disponível em: http://www.cnpq.br/documents/10157/56b578c4-0fd5-4b9f-b82a-e9693e4f69d8 Acessado em: 20/12/2019

MOLLES, M.C.; SHER, A. Ecology: Concepts and Applications. New York, NY: McGraw-Hill Education, p.572, 2019.

NOVO, E.M.L.M.; BARBOSA, C.C.F.; LOBO, F.L. Comportamento espectral de ambientes aquáticos. In: MENESES, P.R.; ALMEIDA, T. BATISTA, G.M.M. Reflectância de materiais terrestres: análise e interpretação. Oficina de Textos, São Paulo - SP, 2019.

NUNES DA CUNHA, C.; JUNK, W. J. Parte II: A Classificação dos Macrohabitats do Pantanal Mato-grossense. In Classificação e delineamento das áreas úmidas brasileiras e de seus macrohabitats. NUNES DA CUNHA, C.; PIEDADE, M. T. F.; JUNK, W. J. eds. Cuiabá: EdUFMT, 2014.

NUNES DA CUNHA, C.; PIEDADE, M.T.F.; JUNK, W.F. Classificação e Delineamento das Áreas Úmidas Brasileiras e de seus Macrohabitats. Instituto Nacional de Ciência e Tecnologia em Áreas Úmidas - INAU. Cuiabá, 2015. EdUFMT. Disponível em: www.INAU.org.br/imprensa/?CategoriaCod=1 Acesso em: $10 / 12 / 2019$

PARANHOS FILHO, A.C.; MOREIRA, E.S.; OLIVEIRA, A.KL. M.; PAGOTTO, T.C.S.; MIOTO, C.L. Análise da variação da cobertura do solo no Pantanal de 2003 a 2010 através de sensoriamento remoto. Engenharia Sanitaria e Ambiental, v.19, n.(spe), p. 69-76, 2014. Disponível em: http://www.scielo.br/scielo.php? pid=S141341522014000500069\&script=sci_arttext\&tlng=pt https://dx.doi.org/10.1590/S1413-41522014019010000305

PURKIS, S.; ROELFSEMA, C. Remote Sensing of Submerged Aquatic Vegetation and Coral Reefs. In: Remote Sensing of Wetlands TINER, R.W.; LANG, M.W.; KLEMAS, V.V. CRC Press: Boca Raton, FL, USA, p.79-118, 2015.

QGIS - DEVELOPMENT TEAM (2016). Um Sistema de Informação Geográfica livre e aberto. Disponível em: https://www.qgis.org/pt_BR/site/. Acesso em: $05 / 02 / 2018$

SALLUN FILHO, W.; KARMANN, I. Geomorphological map of the Serra da Bodoquena karst, west-central Brazil, Journal of Maps, v.3, n.1, p.282-295, 2007. Disponível em: https://www.tandfonline.com/doi/abs/10.1080/jom.2007.9710845. DOI: 10.1080/jom.2007.9710845.

SÁNCHEZ, L.E.; LOBO, H.A.S. (Orgs.), Guia de Boas Práticas Ambientais na Mineração de Calcário em Áreas Cársticas. Sociedade Brasileira de Espeleologia, Campinas, $2016 . \quad$ Disponível em: http://www.terrabrasilis.org.br/ecotecadigital/index.php/estantes/espeleologia/3637guia-de-boas-praticas-ambientais-na-mineracao-de-calcario-em-areas-carsticas Acessado em: 8/01/2020 
SCREMIN-DIAS, E.; POTTI, V.J.; DAMASCENO-JUNIOR, G.A.; DE MEIRA CRISTALDO, A.C.; DIAS, F.A.; POTT, A. Flora de campos úmidos calcários da Serra da Bodoquena. Iheringia. Série Botânica, v.73, p.80-92, 2018. Disponivel em: https://isb.emnuvens.com.br/iheringia/article/viewFile/681/391 Acessado em: 22/06/2019.

SILVA, N.M. da.; PIVELLO, V. R. Conservação dos remanescentes vegetais de cerrado e a dinâmica de uso e ocupação das terras em Bonito, Mato Grosso do Sul. Revista Brasileira de Agroecologia, v.4, n.3, p.86-96, 2009. Disponível em: https:// orgprints.org/25288/ Acessado em: 8/01/2020.

SILVA, T.S.; COSTA, M.P.; MELACK, J.M.; NOVO, E.M. Remote sensing of aquatic vegetation: theory and applications. Environmental Monitoring and Assessment, v.140, p.131-145, 2008. Disponível em: https://link.springer.com/article/10.1007/s10661-007-9855-3 DOI: 10.1007/s10661007-9855-3

TERUYA JUNIOR H.; LASTORIA, G.; CORREA, L.C.; DALMAS, F.B.; PARANHOS FILHO, A.C. Vulnerabilidade Natural e Ambiental da Bacia Hidrográfica Rio Formoso, Mato Grosso do Sul. Anuário do Instituto de Geociências - UFRJ, v.41, n. 2, p.41-50, 2018. Disponível em: http://www.ppegeo.igc.usp.br/index.php/anigeo/article/view/12969/12563 Acessado em: 13/11/2019.

TINER, R.W. Introduction to Wetland Mapping and Its Challenges. In: Remote Sensing of Wetlands TINER, R.W.; LANG, M.W.; KLEMAS, V.V. CRC Press: Boca Raton, FL, USA, p. 63, 2016.

USGS - United States Geological Survey (2018a). Earth Explorer - Home. Disponível em: https://earthexplorer.usgs.gov/. Acesso em: 13/05/2018a

USGS - United States Geological Survey (2018b). USGS EROS Archive - Landsat Archives - Landsat 8 OLI/TIRS Level-2 Data Products - Surface Reflectance. Disponível em: https://www.usgs.gov/media/files/land-surface-reflectance-code-lasrcproduct-guide Acessado em: 13/05/2018b

USGS 1984. United States Geological Survey (2018c). Imagens LANDSAT 4-5 TM. OBITA 226, PONTO 075. Disponível em: https://earthexplorer.usgs.gov/ Acessado em: 13/05/2018c

USGS 1984. United States Geological Survey (2018d). Imagens SENTINEL- 2. QUADRANTE T21KWS. Disponível em: https://earthexplorer.usgs.gov/ Acessado em: $13 / 05 / 2018 d$

USGS - United States Geological Survey (2018e). Shuttle Radar Topography Mission - SRTM. Disponível em: http://srtm.usgs.gov/data/obtainingdata.html. Acesso em: 13/05/2018e

VIEIRA, I.C.G.; TOLEDO, P.M.D.; HIGUCHI, H. A Amazônia no antropoceno. Ciência e Cultura, v.70, n.1, p.56-59, 2018. Disponível em: http://cienciaecultura.bvs.br/scielo.php?pid=S0009-

67252018000100015\&script=sci_arttext\&tlng=en Acessodo em: 10/01/2020. 
WU, Q. GIS and Remote Sensing Applications in Wetland Mapping and Monitoring. In: Huang, B. (Ed.), Comprehensive Geographic Information Systems, Oxford: Elsevier, v. 2, p. 140-157, 2017. DOI: 10.1016/B978-0-12-409548-9.10460-9 Acessado em: 22/12/2019 